via computerized literature searches and cross-referencing from original and review articles. The final reference list was reviewed for completeness by a recognized expert in the area of PE repair. Inclusion criteria incorporated the availability of quantitative measures of preoperative and postoperative cardiovascular function, time between assessments, and description of the procedures used. Reported measures of cardiac function varied between studies and included cardiac output, maximum oxygen uptake, exercise capacity, oxygen pulse, maximum heart rate and stroke volume. For the purpose of the analysis these variables were combined into a single index termed 'cardiovascular function'. In all, 8 studies including a total of 169 patients were identified that fitted the inclusion criteria. Using random-effects modeling, the mean weighted effect size for cardiovascular function after surgical repair of $\mathrm{PE}$ was statistically significant (0.59, 95\% Cl 0.25-0.92; $P=0.0006)$.

The findings of this study contradict arguments that surgical repair of PE is primarily cosmetic, and demonstrate that there are significant improvements in cardiovascular function as a result of this procedure.

Original article Malek MH et al. (2006) Cardiovascular function following surgical repair of pectus excavatum: a metaanalysis. Chest 130: 506-516

\section{Routine use of ramipril for preventing diabetes is not indicated}

Agents that block the renin-angiotensin system have been reported to prevent diabetes. An international team of investigators performed a double-blind, randomized multicenter trial to evaluate the effect of ramipril, an angiotensinconverting-enzyme inhibitor, on the development of diabetes. In total, 5,269 participants aged 30 years or older, with impaired fasting plasma glucose levels or impaired glucose tolerance (i.e. at high risk of developing diabetes) but with no history of cardiovascular disease, were assigned to receive either ramipril or placebo, and were followed up for a median of 3 years.

Ramipril did not significantly decrease the incidence of diabetes or death compared with placebo. The drug did, however, have some beneficial effects on glucose metabolism, raising the rate of regression to normoglycemia $(42.6 \%$ of patients in the ramipril group versus $38.2 \%$ of patients in the placebo group) and improving tolerance of ingested glucose (plasma glucose levels $2 \mathrm{~h}$ after oral glucose administration were $7.5 \mathrm{mmol} / \mathrm{l}$ in the ramipril group and $7.8 \mathrm{mmol} / \mathrm{l}$ in the placebo group). Patients treated with ramipril also experienced a larger drop in alanine aminotransferase levels than those taking placebo (3.4 U// versus 2.3U/I). There were no significant differences between the two groups with regard to frequency of cardiovascular events, creatinine levels and weight gain. The authors conclude that the favorable effects of ramipril on glycemic control during the 3-year study period were not sufficient to indicate its use for the prevention of diabetes.

Original article The DREAM Trial Investigators (2006) Effect of Ramipril on the incidence of diabetes. N Engl J Med 355: 1551-1562

\section{Sirolimus-eluting and paclitaxel- eluting stents for acute myocardial infarction}

The likelihood of restenosis following implantation of a coronary artery stent is lower in some patient subgroups if a drug-eluting-as opposed to an uncoated - device is used; however, few data on drug-eluting stents for acute myocardial infarction (MI) with ST-segment elevation are available. Two recent trials of the main commercially available stents have addressed this issue.

Spaulding and colleagues conducted a prospective, randomized trial to compare sirolimus-eluting stents (CYPHER $^{\circledR}$ or CYPHER SELECT ${ }^{\circledR}$; Cordis Corporation, Miami Lakes, FL) with commercially available uncoated stents, in 712 patients selected from 48 medical centers across 15 countries. Patients-eligible for inclusion if symptoms of their first Ml began within the $12 \mathrm{~h}$ preceding catheterization-were split evenly between the two groups and blinded to treatment. The trial-TYPHOON-was carried out from October 2003 to October 2005. Patients were followed for 12 months.

After 1 year, the treated vessel had failed in $7.3 \%$ of patients who had received a sirolimuseluting stent, compared with $14.3 \%$ of those who had received uncoated stents $(P=0.004)$. 